\title{
Thermal-Hydrogen Promoted Selective Desorption and Enhanced Mobility of Adsorbed Radicals in Silicon Film Growth
}

\author{
S. Cereda, F. Zipoli, M. Bernasconi, Leo Miglio, and F. Montalenti* \\ L-NESS and Dipartimento di Scienza dei Materiali della Università degli Studi di Milano-Bicocca, Via Cozzi 53, I-20125 Milano, Italy
}

(Received 17 July 2007; published 1 February 2008)

\begin{abstract}
Car-Parrinello simulations and static density-functional theory calculations reveal how hydrogen promotes growth of epitaxial, ordered Si films in plasma-enhanced chemical vapor deposition at lowtemperature conditions where the exposed $\mathrm{Si}(001)-(2 \times 1)$ surface is fully hydrogenated. Thermal $\mathrm{H}$ atoms, indeed, are shown to selectively etch adsorbed silyl back to the gas phase or to form adsorbed species which can be easily incorporated into the crystal down to $T \sim 200{ }^{\circ} \mathrm{C}$ and start diffusing around $T \sim 300^{\circ} \mathrm{C}$. Our results are well consistent with earlier experiments.
\end{abstract}

DOI: 10.1103/PhysRevLett.100.046105

PACS numbers: 81.15.Gh, 68.43.Bc, 68.47.Fg

Plasma-enhanced chemical vapor deposition (PECVD) is a very popular technique for growing silicon films. The possibility of reaching high growth rates at moderate temperatures has made PECVD particularly appealing for generating amorphous $(a)$, microcrystalline $(\mu c)$, and nanocrystalline $(n c)$ films, of particular importance, e.g., in the production of solar cells [1]. By reducing the growth rate [2] or by raising the substrate temperature [3] also epitaxial silicon films of good quality can be obtained. This versatility surely makes PECVD of extreme scientific interest. Among the large amount of information coming out of decades of experiments and production, one phenomenon has captured particular attention, leading to several, sometimes controversial, interpretations. Increasing amounts of hydrogen impinging the surface were indeed shown to enhance crystallinity both during growth, and/or in post-growth treatments $[2,4,5]$. The observation of a simultaneous decrease in the growth rate, and/or of the thinning of the samples during hydrogen exposure, strongly suggested hydrogen to be able to preferentially etch noncrystalline regions [2,6]. A further, possibly complementary channel for H-induced crystallinity in postgrowth exposure was also proposed [5]. While very many indications for possible competing mechanisms were given based on experimental observations, the complexity of the problem, in terms of geometry, chemistry, and the multitude of species involved, has prevented so far the development of a parameter-free quantitative modeling of the process at the atomic scale able to shed full light on the phenomenon.

In this Letter we shall focus on the role played by hydrogen during silicon-film growth starting from a fully hydrogenated $\mathrm{Si}(001)-(2 \times 1)$ representative of the initial stages of low-temperature deposition, where thermal $\mathrm{H}$ desorption is not activated [7]. Our study has been motivated by the wide interest in hydrogen-induced crystallization, and, more specifically, by the seminal paper of Tsai et al. [2] in which it is demonstrated the possibility of growing epitaxial Si films with PECVD at a very low temperature $T=200{ }^{\circ} \mathrm{C}$, provided that silane is introduced in the reactor strongly diluted with $\mathrm{H}_{2}$. In their paper, Tsai et al. suggested preferential etching of nonepitaxial adsorbed species to cause the improved quality of the grown sample. Hydrogen etching is proposed to be selective to such an extent as to remove any wrongly located adsorbate, while leaving already epitaxial species at the surface. Based on $a b$ initio calculations here we shall show that etching does indeed play a key role, but through a much richer variety of induced surface phenomena including promotion of epitaxial sites and surface diffusion. As demonstrated in Refs. [8,9], impingement of $\mathrm{SiH}_{3}$, the dominant radical in PECVD under several working conditions $[10,11]$, easily produces a surface dangling bond on $\mathrm{Si}(001)-(2 \times 1): \mathrm{H}$ to which a second incoming silyl molecule can stick forming the stable, but not epitaxial, configuration reported in Fig. 1 (inset 2Ha). We have investigated possible paths out of this state using static density-functional theory calculations with ultrasoft pseudopotentials [12] as implemented in the plane-wave VASP code $[13,14]$. We adopted Perdew and Wang [15] gradient corrected exchange-correlation functional. Spinpolarization was taken into account. We used a slab of 6 layers with 16 atoms per layer and a vacuum region of $\sim 10 \AA$. The surface was passivated by hydrogen (both at the top, in the dimerized configuration, and at the bottom). A $2 \times 2 \times 1$ Monkhorst-Pack $k$-points mesh was used and the energy cutoff was set to $240 \mathrm{eV}$. In geometry optimizations all of the atoms were allowed to relax except for the two bottom Si layers that were kept frozen to bulk positions. Activation energies were computed using the nudged elastic band technique [16] with the climbing image refinement [17]. Convergence at local-minima (saddle points) was achieved when forces were less than $0.005 \mathrm{eV} / \AA$ $(0.02 \mathrm{eV} / \AA)$. Dozens of barriers were sampled in order to understand the full kinetic scenario characterizing possible evolution out of the initial state. The result of these calculations was rather clear: no barrier out of state $2 \mathrm{Ha}$ below $E_{a} \sim 2.2 \mathrm{eV}$ seems to exist. Using harmonic tran- 


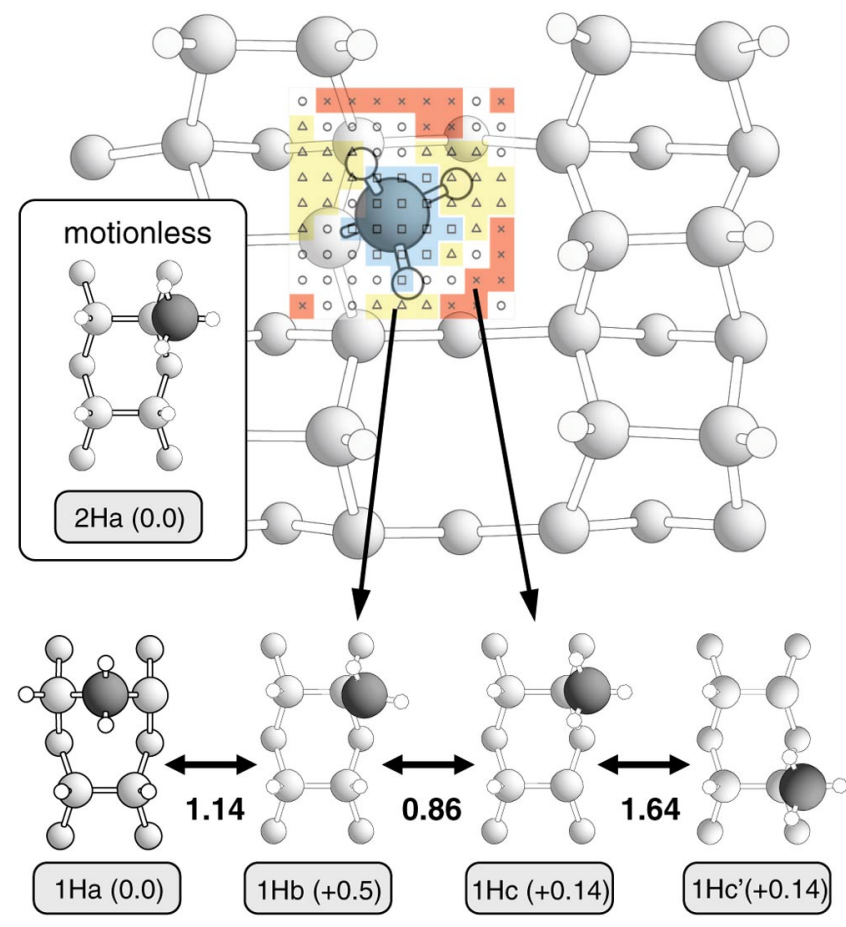

FIG. 1 (color online). Cross section for the processes induced by a $\mathrm{H}$ atom hitting a $\mathrm{SiH}_{3}$ adsorbed on a dangling bond (inset $2 \mathrm{Ha}$ ). The number preceding the minimum label indicates the number of $\mathrm{H}$ atoms in excess with respect to $\mathrm{Si}(001)-(2 \times 1): \mathrm{H}$. Different symbols and different colors (in the online version) are used to distinguish among the mechanisms. $\square$ (light blue): a $\mathrm{SiH}_{4}$ molecule is abstracted by the incoming $\mathrm{H}$. $\triangle$ (yellow): a $\mathrm{SiH}_{2}$ adsorbed on the surface $(1 \mathrm{Hb})$ and a desorbed $\mathrm{H}_{2}$ are produced. $\times$ (red): the incoming $\mathrm{H}$ removes a $\mathrm{H}$ atom from an adjacent dimer leaving a reactive dangling bond $(1 \mathrm{Hc})$. $\bigcirc$ (white): recoil. The area associated with each single point is $0.25 \AA^{2}$. The path for incorporation in the crystal or for diffusion along the dimer row is shown at the bottom. The energies of the displayed minima and of the transition states (in bold) are reported in $\mathrm{eV}$ and are computed relatively to the lowest-energy state with the same number of $\mathrm{H}$ atoms.

sition state theory for calculating the residence time $\tau$,

$$
\tau=\frac{1}{\nu_{0}} \exp \left(E_{a} / k_{B} T\right)
$$

$k_{B}$ being the Boltzmann constant, $T$ the substrate temperature, and $\nu_{0}$ a frequency prefactor (set to the standard value $\nu_{0}=10^{13} \mathrm{~s}^{-1}$ ), for $E_{a}=2.2 \mathrm{eV}$ and $T=200^{\circ} \mathrm{C}$, one obtains $\tau$ of the order of centuries. The system is completely frozen in the initial adsorption site. We have therefore checked what happens if thermal hydrogen hits such motionless silyl. To this goal, we have performed $a b$ initio Car-Parrinello molecular dynamics simulations [18] using the CPMD code [19]. More details on the actual simulation procedure can be found in Ref. [9], where the same parameters were used except for the substrate temperature, here set to $T=200^{\circ} \mathrm{C}$.
After running 81 simulations with atomic $\mathrm{H}$ impinging the substrate with a thermal energy of $0.1 \mathrm{eV}$, we were able to build the cross section displayed in Fig. 1. Events can be divided into three main categories, which occur roughly with the same probability. Hydrogen can etch $\mathrm{SiH}_{3}$ by an Eley-Rideal mechanism coming back to the gas phase as silane (region indicated with boxes in Fig. 1 with a cross section $\sigma \sim 3.8 \AA^{2}$, the sampled surface cell being $20.3 \AA^{2}$ ), it can capture one hydrogen from the adsorbed silyl leaving an adsorbed $\mathrm{SiH}_{2}$ in the configuration $1 \mathrm{Hb}$ displayed in Fig. 1, or, beside simple recoil, it can etch an adjacent surface $\mathrm{H}$ atom leaving a highly reactive dangling bond (for instance, configuration $1 \mathrm{Hc}$ in Fig. 1). These results show that etching is indeed possible with some non-negligible probability. Is it also strongly preferential as suggested in Ref. [2]? To answer this question, we have recomputed the etching cross section when $\mathrm{SiH}_{3}$ is adsorbed in a weaker bound configuration. In particular, we have positioned the adsorbed silyl bound to a silicon atom of the second layer, in the configuration highlighted in Fig. 2. This configuration has been identified as a possible adsorption site from $a b$ initio static calculation [20] and from MD simulations of the impact of $\mathrm{SiH}_{3}$ radicals on $\mathrm{Si}(001)-(2 \times 1): \mathrm{H}$ [9]. The cross section for $\mathrm{H}$-induced etching is reported in Fig. 2: it strongly raises passing from $\sigma \sim 3.8 \AA^{2}$ for the stable configuration (Fig. 1) to a value of $\sigma \sim 8.0 \AA^{2}$, confirming the selectivity of the process as proposed in Ref. [2].

However, if selective etching explains how noncrystalline features can be removed, it is not sufficient to understand how an epitaxial film can actually grow. Indeed, we

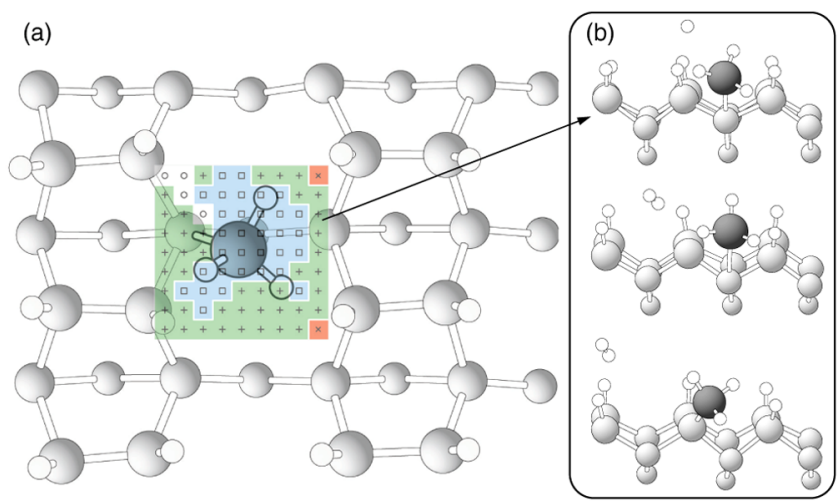

FIG. 2 (color online). (a) Cross section for the processes induced by a $\mathrm{H}$ atom hitting a $\mathrm{SiH}_{3}$ adsorbed on the second layer. $\square$ (light blue): the incoming $\mathrm{H}$ atom induces the $\mathrm{SiH}_{3}$ etching by formation of silane. $\times$ (red): a $\mathrm{H}$ atom from a near dimer is abstracted without affecting the adsorbed $\mathrm{SiH}_{3}$. + (green): $\mathrm{a} \mathrm{SiH}_{3}$ adsorbed on a dangling bond (configuration $2 \mathrm{Ha}$ ) is formed after the abstraction of a $\mathrm{H}$ atom from the adsorbate or from nearest dimers as sketched in panel (b). (white): recoil. (b) From top to bottom: snapshots of a CarParrinello simulation where $\mathrm{SiH}_{3}$ immediately jumps to occupy the newly formed dangling bond. 
still need to explain how incorporation into epitaxial sites is possible. Let us consider the other two products (beside etched silane) of $\mathrm{SiH}_{3}$ interaction with impinging $\mathrm{H}$ (configurations $1 \mathrm{Hb}$ and $1 \mathrm{Hc}$ of Fig. 1). After computing a set of possible barriers out of these states, the following scenario emerged. From configuration $1 \mathrm{Hb}$, the system can incorporate into the crystal by directly jumping to the epitaxial configuration $1 \mathrm{Ha}$ surmounting a barrier of $0.64 \mathrm{eV}$. However, a lower-energy mechanism exists, leading to configuration $1 \mathrm{Hc}$. Considering the relative energy of the three involved local minima (reported in the figure), the effective barrier for reaching the epitaxial site starting from $1 \mathrm{Hb}$ is of $\sim 1 \mathrm{eV}$. Using again Eq. (1) for $T \sim$ $200{ }^{\circ} \mathrm{C}$, one finds $\tau \sim 4.6 \mathrm{~ms}$. At any deposition rate $\Phi \ll$ $1 / \tau \sim 200 \mathrm{ML} / \mathrm{s}$, it is then possible to grow epitaxial films. Considering the very slow growth rate used in Ref. [2], the reported high degree of crystallinity is fully consistent with our findings. The here presented incorporation process is very similar to the one proposed in Ref. [21] (silicon growth) and also in Ref. [22] (diamond growth). Notice, however, that here the whole set of processes leading to final incorporation from the initial radical adsorption site were considered and quantified.

While incorporation is the key step to grow epitaxial films, it is often also desirable to obtain films with small surface roughness. To this goal, fast diffusion of adsorbed species is needed [23,24]. While no detailed information about the roughness of the films grown in Ref. [2] is given, we find it useful and interesting to provide a theoretical investigation of surface mobility which could be exploited in future experiments aiming at controlling both epitaxy and surface corrugation. From site $1 \mathrm{Hc}$, the silyl can diffuse as a whole along the dimer row to configuration $1 \mathrm{Hc}^{\prime}$. While the barrier for this event is of $\sim 1.5 \mathrm{eV}$, actual long-range diffusion would imply visiting again the stablest site, yielding an effective $E_{a} \sim 1.64 \mathrm{eV}$. This value corresponds to a diffusive time $\tau_{D}$ of several hours at $T=$ $200{ }^{\circ} \mathrm{C}$, mobility starting to become relevant at around $400{ }^{\circ} \mathrm{C}\left(\tau_{D} \sim 0.1 \mathrm{~s}\right.$ is here and below taken as an indicative threshold for defining the onset of diffusion, the estimate being relevant for deposition fluxes $\lesssim 10 \mathrm{ML} / \mathrm{s}$ ). However, one should take into account that while the adsorbate is diffusing under actual growth conditions, impinging $\mathrm{H}$ atoms could saturate the dangling bond which is present in any of the visited configurations. This would be detrimental since, as we have shown, no diffusive event takes place in the absence of the dangling bond. In this case (or, more in general, if the temperature is too low for activating even a single of the $1.64 \mathrm{eV}$ jumps), we might ask what happens if a further $\mathrm{H}$ impinges on configuration 1Ha. Following the same procedure used to generate Fig. 1 the cross section illustrated in Fig. 3 is found. The region with extension $\sigma \gtrsim 19.3 \AA^{2}$ marked with upwards triangles indicates where incoming $\mathrm{H}$ removes one of the $\mathrm{SiH}_{2}$ hydrogens or the $\mathrm{H}$ saturating the underlying dimer, leav-

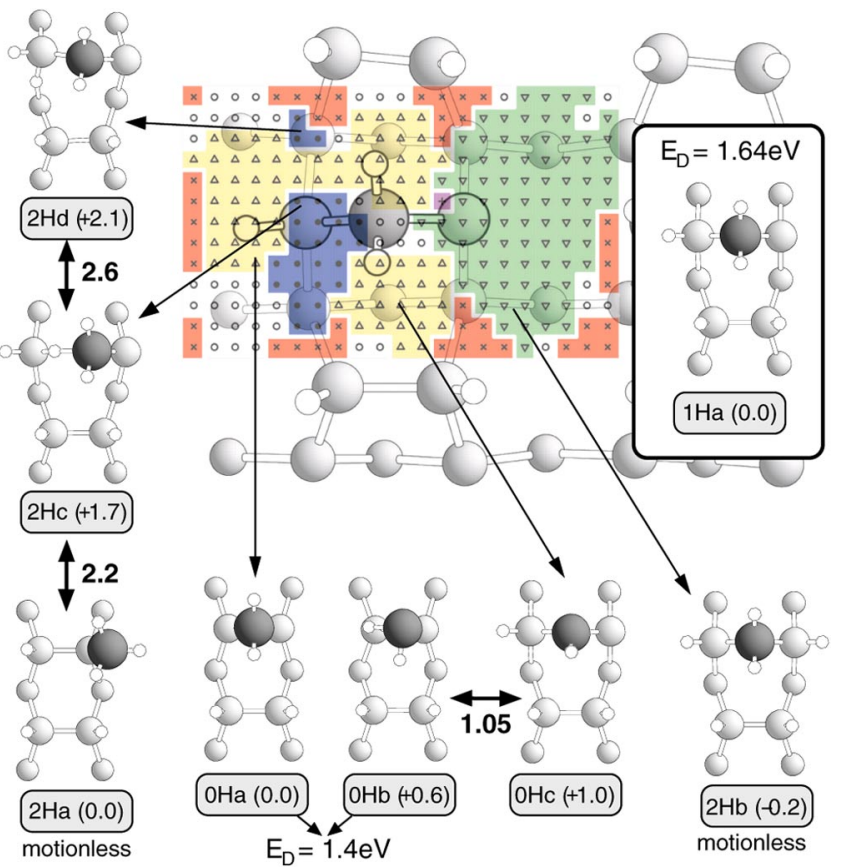

FIG. 3 (color online). Cross section for the processes induced by a $\mathrm{H}$ atom hitting a $\mathrm{SiH}_{2}$ molecule adsorbed in configuration 1Ha (inset). $\triangle$ (yellow): a $\mathrm{H}$ atom is removed forming $\mathrm{H}_{2}$ and the configurations $\mathrm{OHa}$ or $\mathrm{OHc}$, the latter immediately decaying to $0 \mathrm{Hb}$ after surmounting a barrier of $0.05 \mathrm{eV}$. (dark blue): the impinging $\mathrm{H}$ atom breaks a $\mathrm{Si}-\mathrm{Si}$ bond forming configuration $2 \mathrm{Hc}$ (which can evolve to $2 \mathrm{Ha}$ surmounting a barrier of only $\sim 0.5 \mathrm{eV}$ ) or $2 \mathrm{Hd}$. $\nabla$ (green): configuration $2 \mathrm{Hb}$ is obtained due to the adsorption of incoming $\mathrm{H}$ on the dangling bond. $\times$ (red): a $\mathrm{H}$ atom of the neighboring dimers is abstracted by the incoming H. $\bigcirc$ (white): recoil. + (purple) corresponds to a simulation where configuration $2 \mathrm{Ha}$ was directly reached in dynamics. The energies of minima and transition states (in bold) are indicated with respect to the corresponding lowest state, as in Fig. 1.

ing the substrate as $\mathrm{H}_{2}$ and creating the configurations $0 \mathrm{Ha}$ or $0 \mathrm{Hc}$ (Fig. 3). The latter evolves into $0 \mathrm{Hb}$ almost immediately, since a barrier of only $0.05 \mathrm{eV}$ must be surmounted. A quick comparison with Ref. [25] reveals $0 \mathrm{Ha}$ and $\mathrm{OHb}$ to be two of the local minima visited by a $\mathrm{Si}$ adatom when diffusing on $\mathrm{Si}(001)-(2 \times 1): \mathrm{H}$. This means that $\mathrm{H}$-induced removal of $1 \mathrm{H}$ effectively transforms the adsorbed $\mathrm{SiH}_{2}$ in $1 \mathrm{Ha}$ into a simple adatom diffusing on a fully hydrogenated environment. As demonstrated in Ref. [25] using the local density approximation, such an adatom is very mobile, since an effective barrier $E_{D} \sim$ $1.2 \mathrm{eV}$ characterizes diffusion along dimer rows. By repeating the calculation along the full path proposed in [25] with our gradient corrected exchange-correlation functional approach, we find only a slightly higher estimate $E_{D} \sim 1.4 \mathrm{eV}$. In the present contest, two important conclusions can be made. First, thanks to the etching process, diffusion down to $T \sim 300^{\circ} \mathrm{C}$ is allowed. Moreover, the whole diffusive process does not need additional freeing of dangling bonds in the neighborhood of the moving Si atom. 
Therefore, surface diffusion can be sustained along extended paths also in the presence of continuously incoming $\mathrm{H}$. Let us now analyze the remaining simulations. When $\mathrm{H}$ sticks in the left region of the sampled impact area of Fig. 3 configuration $2 \mathrm{Hc}$ (or more rarely $2 \mathrm{Hd}$ ) is obtained, which can evolve with a barrier of $\sim 0.5 \mathrm{eV}$ to the starting and motionless configuration $2 \mathrm{Ha}$, ready to be further on etched. Alternatively, the epitaxial minimum $2 \mathrm{Hb}$ displayed in Fig. 3 is reached (when $\mathrm{H}$ sticks on the right region of the impact area and saturates the dangling bond). Here the adsorbed radical, while perfectly epitaxial, is totally trapped (see also Ref. [21]) so that it could be interesting to monitor its behavior under further $\mathrm{H}$ impingement. This does not require further simulations, since, due to the close resemblance with configuration 1Ha we can exploit the left portion of the cross section of Fig. 3. Three different outcomes are thus possible: one of the $\mathrm{H}$ atoms saturating the dangling bond of the same $\mathrm{Si}$ atom to which $\mathrm{SiH}_{2}$ is bound can be etched, restoring configuration $1 \mathrm{Ha}$; alternatively, one of the $2 \mathrm{H}$ atoms of $\mathrm{SiH}_{2}$ is etched, or, the incoming $\mathrm{H}$ atom is inserted into the $\mathrm{Si}-\mathrm{Si}$ bond, similarly to configuration $2 \mathrm{Hc}$ of Fig. 3. While we have not completely explored possible further evolution, we believe none of the above possibilities to open up channels for faster mobility.

Hydrogen-induced enhanced mobility of ad-species was invoked in Ref. [26] to explain the experimentally observed strong decrease in film roughness with increasing $\mathrm{H}$ pressure. Although it is tempting to relate our theoretical results also with this evidence, we note that in such work $a$ and $\mu c$ films were considered, so that the barriers here computed for the crystalline (001) surface should not be considered as more than indicative. A more direct confirmation of the predictive power of our calculations can be perhaps found in the etching experiments of Ref. [27], where both the here-presented $\mathrm{H}$-induced mechanisms (silyl removal as silane, $\mathrm{H}_{2}$ removal leaving adsorbed $\mathrm{SiH}_{2}$ ) were clearly identified. A further study of silyl etching by atomic hydrogen is described in Ref. [28]. The there observed slow decay of the etching rate with decreasing temperature seems fully compatible with our estimation of $\sim 0.05 \mathrm{eV}$, obtained performing a nudged elastic band calculation with the same procedure followed for computing the diffusion paths.

In summary, we have shown that hydrogen can promote ordered growth of fully hydrogenated PECVD silicon thin films through several channels, including etchingpromoted radical incorporation into epitaxial sites (activated around $T \sim 200^{\circ} \mathrm{C}$ ), etching-promoted diffusion $\left(T \gtrsim 300^{\circ} \mathrm{C}\right)$, and strongly selective etching of metastable adsorption sites. Our prediction for the onset of epitaxy, based on density functional theory, is consistent with experiments. Because of the complexity of the growth process a closer comparison with experiments, e.g., in terms of growth rates and/or surface roughness, still demands for further calculations and extensive modeling. We believe that the identification of elementary processes along with their activation energies and/or cross sections as presented here is of great value for the development of comprehensive kinetic Monte Carlo schemes, strongly needed to close the gap between atomic-scale and macroscopic measurements and gain a full understanding of PECVD growth.

We acknowledge useful discussions with $\mathrm{M}$. Moseler, G. Isella, M. Ceriotti, C. Cavallotti, and funding under the European STREP Project "NANOPHOTO" and by the Cariplo Foundation (SIMBAD Project). Computational support from CILEA supercomputing facilities and the CINECA (CNISM Project "Iniziativa Calcolo Parallelo 2006 and 2007") is also acknowledged.

*francesco.montalenti@unimib.it

[1] A. Shah et al., Science 285, 692 (1999).

[2] C. C. Tsai et al., J. Non-Cryst. Solids 114, 151 (1989).

[3] C. Rosenblad et al., J. Vac. Sci. Technol. A 16, 2785 (1998).

[4] A. Matsuda, Jpn. J. Appl. Phys. 43, 7909 (2004).

[5] S. Sriraman et al., Nature (London) 418, 62 (2002).

[6] I. Solomon et al., J. Non-Cryst. Solids 164-166, 989 (1993).

[7] S. Inanaga et al., J. Vac. Sci. Technol. A 23, 1471 (2005).

[8] S. Ramalingam et al., Surf. Sci. 418, L8 (1998).

[9] S. Cereda et al., Phys. Rev. B 75, 235311 (2007).

[10] A. Matsuda, Jpn. J. Appl. Phys. 43, 7909 (2004).

[11] A. Matsuda and K. Tanaka, J. Appl. Phys. 60, 2351 (1986).

[12] D. Vanderbilt, Phys. Rev. B 41, 7892 (1990).

[13] G. Kresse and J. Hafner, Phys. Rev. B 49, 14251 (1994).

[14] G. Kresse and J. Furthmüller, Comput. Mater. Sci. 6, 15 (1996).

[15] J. P. Perdew et al., Phys. Rev. B 46, 6671 (1992).

[16] G. Henkelman, and H. Jónsson, J. Chem. Phys. 113, 9978 (2000).

[17] G. Henkelman et al., J. Chem. Phys. 113, 9901 (2000).

[18] R. Car and M. Parrinello, Phys. Rev. Lett. 55, 2471 (1985).

[19] Copyright IBM Corp. 1990-2003, Copyright MPI für Festkörperforschung Stuttgart 1997-2000, http:// www.cpmd.org.

[20] M. S. Valipa et al., Phys. Rev. Lett. 95, 216102 (2005).

[21] J. K. Kang and C. B. Musgrave, Phys. Rev. B 64, 245330 (2001).

[22] S. J. Harris and D. G. Goodwin, J. Phys. Chem. 97, 23 (1993).

[23] T. Michely and J. Krug, in Islands, Mounds and Atoms (Springer, New York, 2004).

[24] Z. Zhang and M. G. Lagally, Science 276, 377 (1997).

[25] T. Hashizume et al., Appl. Surf. Sci. 216, 15 (2003).

[26] J.E. Gerbi and J.R. Abelson, J. Appl. Phys. 89, 1463 (2001).

[27] A. Dinger et al., Chem. Phys. Lett. 320, 405 (2000).

[28] C. Lutterloh et al., Surf. Sci. 498, 123 (2002). 